\title{
BMJ
}

\section{Bevacizumab for neovascular age related macular degeneration ( $A B C$ Trial): multicentre randomised double masked study}

\begin{abstract}
Adnan Tufail, consultant ophthalmologist, medical retina department, 1 Praveen J Patel, locum consultant ophthalmologist, ${ }^{1}$ Catherine Egan, consultant ophthalmologist, ${ }^{1}$ Philip Hykin, consultant ophthalmologist, Lyndon da Cruz, consultant ophthalmologist, 'Zdenek Gregor, consultant ophthalmologist, ${ }^{1}$ Jonathan Dowler, consultant ophthalmologist, ${ }^{1}$ Mohammed A Majid, consultant ophthalmologist, ${ }^{2}$ Clare Bailey, consultant ophthalmologist, ${ }^{2}$ Quresh Mohamed, consultant ophthalmologist, ${ }^{3}$ Robert Johnston, consultant ophthalmologist, ${ }^{3}$ Catey Bunce, senior statistician, research and development department, ${ }^{1}$ Wen Xing, statistician, research and development department, ${ }^{1}$ for the ABC Trial Investigators
\end{abstract}

\footnotetext{
${ }^{1}$ Moorfields Eye Hospital NHS Foundation Trust, London EC1V 2PD

${ }^{2}$ Bristol Eye Hospital, Bristol, BSL $2 \mathrm{LX}$

${ }^{3}$ Gloucestershire Eye Department, Cheltenham General Hospital, Cheltenham GL53 7AN

Correspondence to: A Tufail adnan.tufail@moorfields.nhs.uk

Cite this as: BMJ 2010;340:c2459 doi:10.1136/bmj.c2459
}

\section{ABSTRACT}

Objectives To evaluate the efficacy and safety of intravitreous bevacizumab injections for the treatment of neovascular age related macular degeneration.

Design Prospective, double masked, multicentre, randomised controlled trial.

Setting Three ophthalmology centres in the United Kingdom.

Participants 131 patients (mean age 81) with wet age related macular degeneration randomised $1: 1$ to intervention or control.

Interventions Intravitreous bevacizumab $(1.25 \mathrm{mg}$, three loading injections at six week intervals followed by further treatment if required at six week intervals) or standard treatment available at the start of the trial (photodynamic treatment with verteporfin for predominantly classic type neovascular age related macular degeneration, or intravitreal pegaptanib or sham treatment for occult or minimally classic type neovascular age related macular degeneration).

Main outcome measures Primary outcome: proportion of patients gaining $\geq 15$ letters of visual acuity at one year ( 54 weeks). Secondary outcomes: proportion of patients with stable vision and mean change in visual acuity. Results Of the 131 patients enrolled in the trial, five patients did not complete the study because of adverse events, loss to follow-up, or death. In the bevacizumab group, 21 (32\%) patients gained 15 or more letters from baseline visual acuity compared with two (3\%) in the standard care group ( $\mathrm{P}<0.001)$; the estimated adjusted odds ratio was 18.1 (95\% confidence interval 3.6 to 91.2 ) and the number needed to treat was 4 ( 3 to 6 ). In addition, the proportion of patients who lost fewer than 15 letters of visual acuity from baseline was significantly greater among those receiving bevacizumab treatment (91\% (59) $v 67 \%$ (44) in standard care group; $\mathrm{P}<0.001$ ). Mean visual acuity increased by 7.0 letters in the bevacizumab group with a median of seven injections compared with a decrease of 9.4 letters in the standard care group ( $\mathrm{P}<0.001)$, and the initial improvement at week 18 (plus 6.6 letters) was sustained to week 54. Among 65 patients treated with bevacizumab, there were no cases of endophthalmitis or serious uveitis related to the intervention. All end points with respect to visual acuity in the study eye at 54 weeks favoured bevacizumab treatment over standard care.

Conclusions Bevacizumab 1.25 mg intavitreous injections given as part of a six weekly variable retreatment regimen is superior to standard care (pegaptanib sodium, verteporfin, sham), with low rates of serious ocular adverse events. Treatment improved visual acuity on average at 54 weeks.

Trial registration number Current controlled trials ISRCTN83325075

\section{INTRODUCTION}

Age related macular degeneration (AMD) is the leading cause of severe visual loss in patients over the age of 50 in Europe and North America. ${ }^{1}$ Phenotypically it can be classified into early and late stages based on specific clinical features. Early age related macular degeneration is characterised by the presence of drusen or pigmentary changes, or both, and is compatible with reasonable vision. A few patients with early age related macular degeneration can progress to develop the late "wet" or neovascular form. Neovascular AMD is characterised by choroidal neovascularisation, which is the growth of abnormal choroidal blood vessels beneath the macula, which causes severe loss of vision and is responsible for most loss of vision caused by age related macular degeneration. ${ }^{2}$

One of the key mediators implicated in the pathogenesis of choroidal neovascularisation in neovascular AMD is vascular endothelial growth factor-A (VEGF). New treatments that target this growth factor are administered by injection into the vitreous cavity of agents 
with high binding specificity. Three agents (pegaptanib (Macugen), ranibizumab (Lucentis), and bevacizumab (Avastin)) that block the action of some or all of the vascular endothelial growth factor-A isoforms are currently in clinical use. They are administered by intraocular (intravitreal) injections with repeat injections as necessary every four to six weeks, depending on the agent. Injections are given through the surface of the eye and delivered into the vitreous cavity, after the instillation of topical anaesthetic and a drop of antiseptic on the eye, and are generally well tolerated and can be given repeatedly. Although the injection itself is relatively painless, the eye is often uncomfortable and gritty for a short period after the procedure.

In 2005 , photodynamic therapy with verteporfin ${ }^{34}$ and intravitreal pegaptanib sodium ${ }^{5}$ were the only treatments approved by the Food and Drug Administration (FDA) and the European Agency for the Evaluation of Medicinal Products (EMEA) for the treatment of neovascular AMD. Although both these treatments are moderately effective at preventing further vision loss, neither results in clinically significant recovery of visual acuity in most patients treated (only about $6 \%$ of treated patients improved vision by 15 letters or more at one year follow-up). ${ }^{45}$ Bevacizumab is a full length monoclonal antibody that binds to and blocks the action of all isoforms of vascular endothelial growth factor. It was initially developed as an intravenous agent in the treatment of metastatic colorectal cancer. ${ }^{6}$ Ophthalmologists then reported promising results with bevacizumab as an intravitreous treatment for neovascular AMD in case series. ${ }^{7-9}$ This treatment has gained popularity worldwide, initially because of perceived superior effectiveness to licensed alternatives in 2006 . It is now probably the most widely used agent to treat neovascular AMD, despite the subsequent licensing of ranibizumab, because of the low cost of treatment when it is used as an intraocular agent. This is particularly the case for developing countries, in which the high unit cost of ranibizumab over bevacizumab has limited its use after licensing. Even in the US, practice pattern reports from the American Academy of Ophthalmology and the American Association of Retina Specialists suggest that most US patients receive bevacizumab rather than ranibizumab for the treatment of neovascular AMD.

Ranibizumab is an antibody fragment with increased binding affinity for all isoforms of vascular endothelial growth factor. It has been shown to be more effective than sham treatment (placebo) in treating minimally classic or occult choroidal neovascularisation ${ }^{10}$ and more effective than photodynamic therapy in treating predominantly classic choroidal neovascularisation. ${ }^{11}$ Patients treated with monthly intravitreal injections of ranibizumab for one to two years showed better visual acuity outcomes than patients treated with sham or photodynamic therapy $(33-40 \%$ of patients treated with ranibizumab improved visual acuity by 15 letters or more compared with $4 \%$ of those treated with placebo and $6 \%$ of those treated with photodynamic therapy). ${ }^{10}{ }^{11}$ Ranibizumab was licensed for use in the treatment of neovascular AMD after we started recruitment for this study and was not routinely funded in England and Wales (National Institute for Health and Clinical Excellence (NICE) approved) until two months before the last patient completed their follow-up and so was not included as a comparator arm.

A unique situation now exists where the licensed intervention (ranibizumab), with level 1 evidence, is used less than a treatment with limited data on efficacy and safety (bevacizumab). Recent review articles on bevacizumab for neovascular AMD highlight the lack of masked trials, the best evidence to date being level $2 \mathrm{~b}$, and the need for level 1 evidence. ${ }^{12} 13$

The current trial (Avastin (bevacizumab) for choroidal neovascular age related macular degeneration (ABC) trial) was designed to investigate whether intravitreal bevacizumab injections are an effective and safe treatment for neovascular AMD compared with standard treatment available on the National Health Service (NHS) in the United Kingdom when we started the trial in 2006. This study is important given the predicted increase in the numbers of patients with neovascular AMD because of increases in life expectancy and the limited healthcare budgets globally. A negative result would lead to cessation of use of an ineffective treatment for the most common cause of loss of vision in elderly people. A positive result would provide the first level 1 evidence for the use of bevacizumab in most people with neovascular AMD who will never have the opportunity to receive ranibizumab because of cost.

\section{METHOD}

\section{Participants}

Participants had to be aged at least 50 ; have a lesion in the study eye with a total size of less than 12 optic disc areas for minimally classic or occult lesions but no more than $5400 \mu \mathrm{m}$ in greatest linear dimension for predominantly classic lesions; have best corrected visual acuity of 6/12 to approximately 6/96 (Snellen equivalent), assessed with the use of charts from the Early Treatment Diabetic Retinopathy Study (ETDRS) (70 to 25 ETDRS $1 \mathrm{~m}$ equivalent letter scores; patients initially view the charts at a starting distance of $4 \mathrm{~m}$, the number of correctly read letters are given a correction factor with the final letter score being the equivalent of a patient reading it at $1 \mathrm{~m}$. A score of 55 letters approximates to 6/24 Snellen acuity); have no permanent structural damage to the central fovea; and have had no previous treatment for exudative age related macular degeneration. Patients were excluded if there was a history of cardiovascular events (myocardial infarction, unstable angina) or cerebrovascular event in the preceding six months. Full inclusion and exclusion criteria have previously been published. ${ }^{14}$ All participants attended the clinic every six weeks for assessment and treatment if required. 


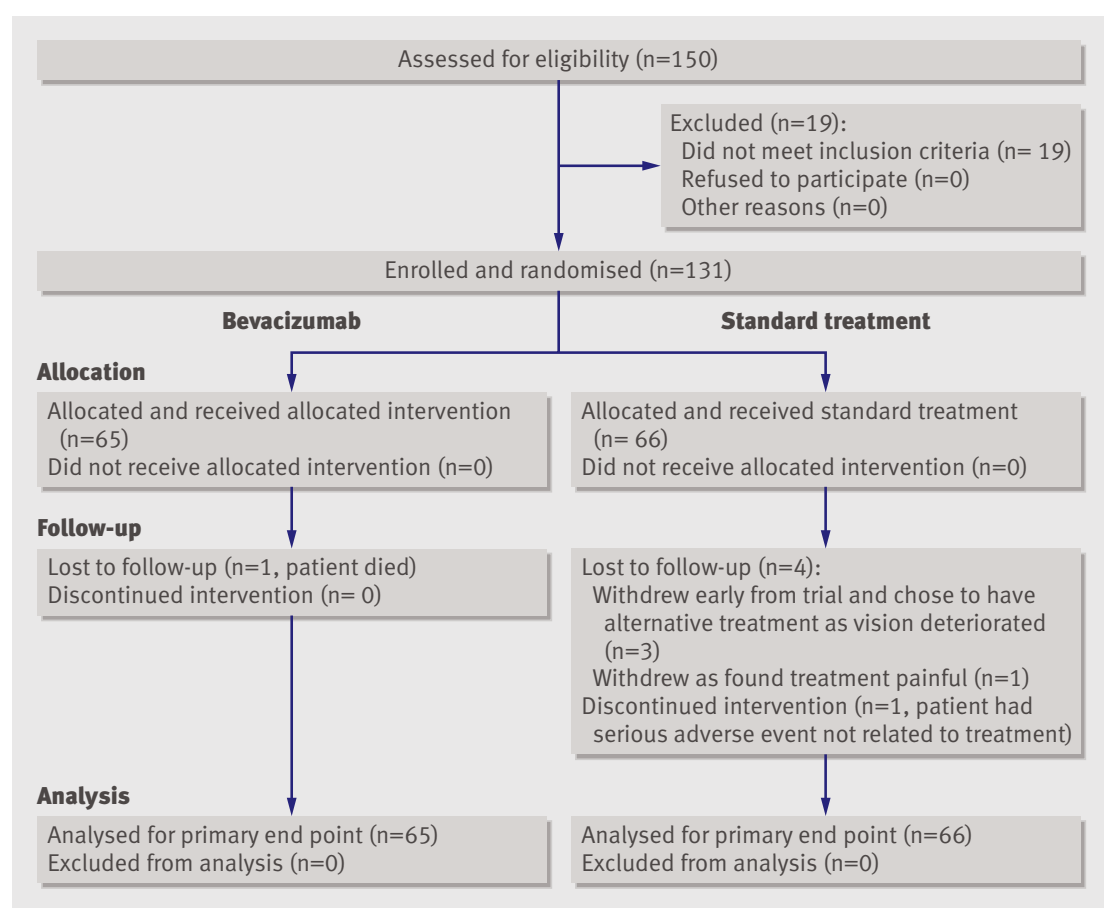

Fig 1 | Details of allocation and flow of patients through trial

\section{Study design}

We carried out a double masked randomised controlled trial with two parallel treatment groups. Eligible patients were randomised in a 1:1 ratio to receive either intravitreal bevacizumab or the standard NHS care available in 2006 (either photodynamic therapy, pegaptanib intravitreal injections) or sham intravitreal injections (placebo). Only one eye per patient was included in the study, and this was selected before randomisation; the trial manager telephoned the clinical trials unit to obtain a treatment allocation. The type of standard care that patients would normally receive was determined before enrolment in the trial, at which point they were allocated to treatment groups by minimisation - a dynamic process that reduced the imbalance between trial arms with respect to eligibility for standard treatment and site.

During recruitment, only patients with well defined neovascular AMD (classic-no occult or predominantly classic choroidal neovascularisation) were eligible for funding for photodynamic therapy in line with NICE guidance. There was no national funding in place for patients with occult-no classic and the minimally classic forms of neovascular AMD (minimally classic or occult choroidal neovascularisation) because photodynamic therapy had not been shown to be effective and pegaptanib sodium had not been appraised by NICE, though it was a licensed treatment. Ranibizumab became licensed only after we had started recruitment and was not approved by NICE until about two months before our final follow-up. At the time of our recruitment, NHS treatment was considered on a case by case basis, and many patients were deemed ineligible for NHS funding. In our trial, however, we included patients with minimally classic or "occult-no classic" choroidal neovascularisation who fulfilled other eligibility criteria for inclusion; they were randomised to standard care (pegaptanib or placebo, based on NHS funding of pegaptanib) or bevacizumab. Figure 1 summarises the enrolment and randomisation process, which has been previously described in detail elsewhere. ${ }^{14}$

During the later stages of recruitment, after ranibizumab had been introduced into clinical practice, we excluded patients who received funding for ranibizumab from enrolment, and patients already recruited were informed of the availability of the newly licensed treatment. At the time of recruitment for this trial, as many patients who were refused public funding of treatment did not have medical insurance and could not afford the costs of private treatment, a considerable proportion would have remained untreated and therefore were happy to consider involvement in a trial in which they were randomised to active treatment (intravitreal bevacizumab) or placebo. Previous precedents for trials with active treatment versus placebo or no treatment included the pegaptanib VISION trial ${ }^{5}$ or the Lucentis MARINA trial. ${ }^{10}$ We fully recruited participants and completed the final follow-up of most of them before final NICE guidance was issued on the use of pegaptanib or ranibizumab for treating neovascular AMD on the NHS.

\section{End points}

Our primary outcome was the proportion of patients gaining 15 or more letters of visual acuity at one year ( 54 weeks); secondary outcomes included the proportion of patients with stable vision and the mean change in visual acuity. ${ }^{14}$ The primary outcome was assessed with an ETDRS visual acuity chart. A gain of 15 letters means being able to read three more lines on the chart; this improvement is equivalent to the smallest letter size read on the test chart being half the size they could read before this magnitude of visual gain.

\section{Active intervention}

Bevacizumab (1.25 mg in $0.05 \mathrm{ml}$ per injection) was administered as intravitreal injections once every six weeks. Each injection was prepared in single use sterile plastic syringes with a six week shelf life and placed in a sealed plastic pouch. After the first three injections, investigators masked to treatment allocation used standardised criteria to decide whether to give further injections, reflecting the real world use of this agent at the time of the trial. The criteria for further treatment were persistence or recurrence of subretinal fluid on optical coherence tomography imaging (Stratus OCT; Carl Zeiss Meditec, Dublin, CA), new haemorrhage, new classic choroidal neovascularisation, or drop of vision by five of more letters with new intraretinal fluid. Patients could therefore receive between three and nine injections over a total of 54 weeks. If there was persistent intraretinal fluid after two consecutive treatments, then treatment was withheld assuming other criteria for retreatment were not triggered 
Table 1|Baseline characteristics of patients and choroidal neovascularisation lesions according to allocated treatment. Figures are percentages (numbers) of participants unless specified otherwise

\begin{tabular}{|c|c|c|}
\hline & $\begin{array}{l}\text { Standard therapy } \\
\qquad(n=66)^{\star}\end{array}$ & $\begin{array}{c}\text { Bevacizumab } \\
\quad(n=65)\end{array}$ \\
\hline Men & $38(25)$ & $40(26)$ \\
\hline Women & $62(41)$ & 60 (39) \\
\hline \multicolumn{3}{|l|}{ Ethnicity (determined by investigators): } \\
\hline White & $99(65)$ & $95(62)$ \\
\hline Other & $2(1)$ & $5(3)$ \\
\hline \multicolumn{3}{|l|}{ Age group (years): } \\
\hline $50-64$ & $2(1)$ & $5(3)$ \\
\hline $65-74$ & $15(10)$ & $22(14)$ \\
\hline $75-84$ & $52(34)$ & $43(38)$ \\
\hline$\geq 85$ & $32(21)$ & $31(20)$ \\
\hline Mean & 81 & 79 \\
\hline \multicolumn{3}{|l|}{ ETDRS visual acuity score $†$ in letters: } \\
\hline Median (IQR) & $53(47-60)$ & $50(43-61)$ \\
\hline$<55$ & $52(34)$ & $57(37)$ \\
\hline$\geq 55$ & $49(32)$ & $43(28)$ \\
\hline \multicolumn{3}{|l|}{ Type of choroidal neovascularisation: } \\
\hline Minimally classic-occult & $76(50)$ & $75(49)$ \\
\hline Predominantly classic & $24(16)$ & $25(16)$ \\
\hline Median (IQR) central $1 \mathrm{~mm}$ macular thickness on OCT & $330(256-359)$ & $328(271-376)$ \\
\hline Median (IQR) total size of lesion $\left(\mathrm{mm}^{2}\right)$ & $6.8(3.1-11.6)$ & $5.4(3.7-11.9)$ \\
\hline Median (IQR) size of choroidal neovascularisation $\left(\mathrm{mm}^{2}\right)$ & $3.3(1.2-7.2)$ & $3.7(1.7-7.3)$ \\
\hline
\end{tabular}

$\mathrm{IQR}=$ interquartile range; $\mathrm{OCT}=$ optical coherence tomography.

*Pegabtanib $n=38$, verteporfin $n=16$, sham $n=12$.

†Visual acuity measured with ETDRS (Early Treatment Diabetic Retinopathy Study) charts at starting distance of $4 \mathrm{~m}$. Score of 55 letters is about equivalent to Snellen $6 / 24$

and there were no signs of choroidal neovascularisation activity.

\section{Control interventions}

Intravitreal pegaptanib injections were given according to the product licence with a dose of $0.3 \mathrm{mg}$ in $0.09 \mathrm{ml}$ by intravitreal injection every six weeks for a year (nine injections in 54 weeks). This reflects the dosing and frequency of treatment used in the pivotal VISION randomised controlled trial. ${ }^{5}$

Verteporfin photodynamic therapy or placebo photodynamic therapy-Verteporfin was administered intravenously and followed by laser irradiation of the macula, according to manufacturer's instructions (www.visudyne.com). ${ }^{3}$ Participants received initial treatment at baseline, with further treatment based on criteria outlined in the pivotal phase III studies.

\section{Measures to maintain masking}

We ensured outcome assessors were masked to treatment allocation by the use of a standard operating protocol that kept the outcome assessors out of contact with treating physicians and unable to obtain access to the treatment allocation, which was kept securely in the clinical trials unit at Moorfields Eye Hospital.

As the analysis of patients randomised to bevacizumab or pegaptanib involved treatment as needed (bevacizumab) compared with a treatment given continuously (nine, six weekly intravitreal injections of pegaptanib), to maintain masking, patients randomised to bevacizumab received sham treatments if they did not require intravitreal treatment at that visit (weeks 18 to 48), according to standardised criteria for retreatment.

Placebo photodynamic therapy-Participants who were randomised to bevacizumab in whom the usual treatment would have been photodynamic therapy (that is, classic-no occult or predominantly classic choroidal neovascularisation) received placebo photodynamic therapy. The procedure was identical to that used for active verteporfin photodynamic therapy except 5\% dextrose was used as placebo with no verteporfin added, as in previous studies. ${ }^{3}$ Care was taken to ensure that the intravenous infusion pump and line were covered as the active verteporfin solution is green while the placebo infusion is a clear solution.

Additional use of sham injections - In line with previous randomised controlled trials, ${ }^{510}$ we used sham rather than placebo intravitreal injections. Sham injections were performed by following the procedure used to prepare the eye for injection but instead of an intravitreal injection, the hub of an empty $1 \mathrm{ml}$ syringe was applied firmly to the conjunctiva to mimic an active injection.

\section{Assessments}

Patients underwent regular assessments of visual acuity and retinal structure (optical coherence tomography imaging) and adverse event reporting at each visit. In addition other functional and structural assessments (including contrast sensitivity measurement and fluorescein angiography) were carried out at predefined time points in the study, as previously described in detail. $^{14}$

\section{Statistical analysis}

Sample size-We determined the number of patients required for significance on the basis of a 1:1 randomisation ratio, with Fisher's exact test for the primary end point and a 0.050 two sided level of significance. We estimated that a sample of 130 patients would have power of $82 \%$ to detect a significant difference between the bevacizumab group and standard care group in gaining 15 or more letters at 54 weeks, assuming a rate of $25 \%$ in the bevacizumab group and $6 \%$ in the standard care group. The proportions used in the power calculations were based on estimates from previous presentations or published studies on bevacizumab, ${ }^{89}$ verteporfin photodynamic therapy, ${ }^{3}$ and pegaptanib. ${ }^{5}$ This number also allowed for a potential $12 \%$ loss to follow-up, estimated from previous clinical trials in such patients. ${ }^{311}$

Statistical analysis - We compared baseline characteristics between the treatment groups to assess the adequacy of randomisation. The proportion of patients who gained 15 letters or more in each group at 54 weeks is shown with 95\% confidence intervals computed by the exact binomial method. We used Fisher's exact test to assess whether or not the observed 

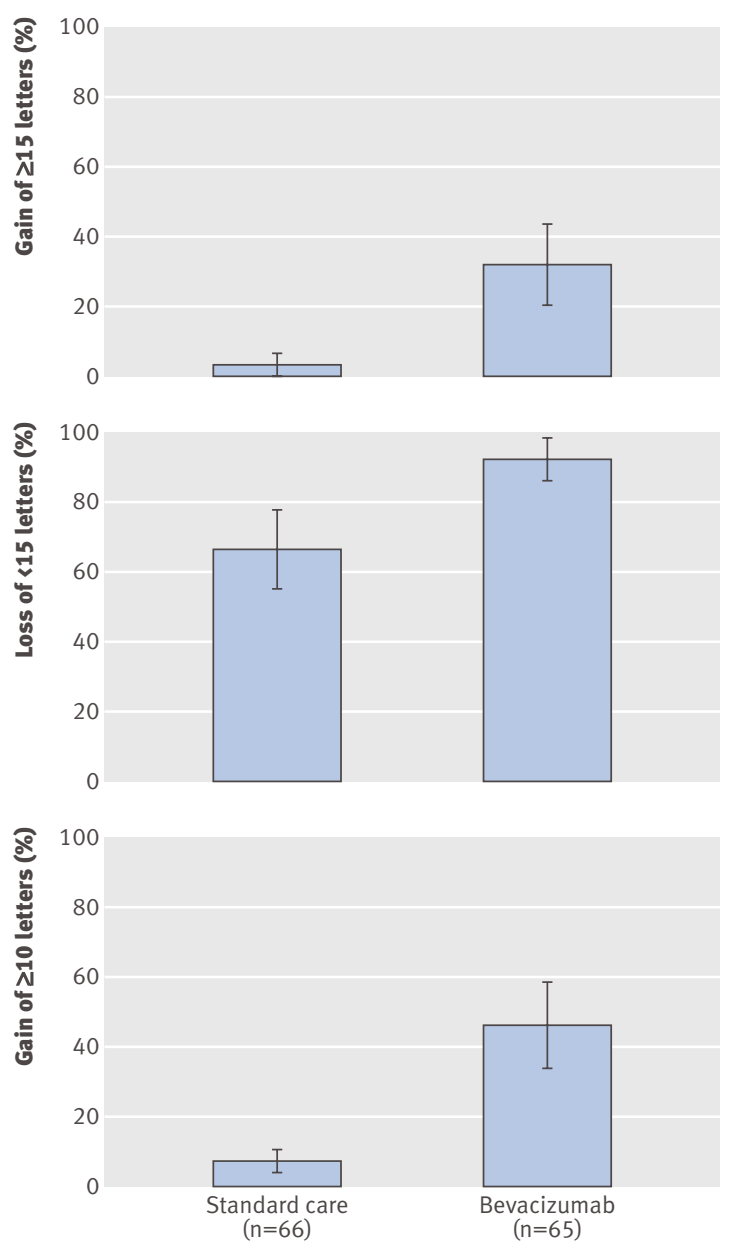

Fig 2 | Summary of results from baseline to 54 weeks according to allocation to standard care or bevacizumab: gain of $\geq 15$ letters (moderate gain) (primary efficacy end point); loss of $<15$ letters (less than moderate vision loss); and gain of $\geq 10$ letters. Confidence intervals based on normal approximation and their standard errors of proportion. Last observation carried forward used to impute missing data. All statistical tests were two sided. P<0.001 for all comparisons of bevacizumab with standard care

differences in proportions were significant. Odds ratios are reported with 95\% confidence intervals to illustrate the contrast between bevacizumab and standard care groups. Patients were analysed in the groups to which they were randomised (intention to treat analysis). Outcome data at 54 weeks were not available for five participants (one allocated to bevacizumab, four allocated to standard care). For the primary analysis and key secondary analyses we carried out an intention to treat analysis (using the last observation carried forward for missing data). In addition, we also analysed the primary end point based on available data from participants who completed the trial. Safety analyses included all treated patients. Odds ratios with 95\% confidence intervals are provided for several secondary outcomes: a gain of 10 letters or more and a loss of more than 15 letters. Summary statistics are reported for the average change from baseline in macular thickness determined by optical coherence tomography and best corrected visual acuity at 54 weeks.

\section{RESULTS}

From August 2006 to November 2007 we enrolled and randomly allocated 131 patients $(65$ eyes of 65 patients to bevacizumab and 66 eyes of 66 patients to standard care (fig 1). The last patient completed one year ( 54 week) follow-up in November 2008. Table 1 summarises the baseline characteristics of patients and choroidal neovascularisation lesions. Most patients were elderly (mean age 81) and white.

More than $90 \%$ of patients in each group (overall $96 \%$ ) were receiving treatment at the last treatment visit (week 48) and were followed up to week 54 . Patients received a mean of 7.1 (median 7, range 3-9) injections of bevacizumab, of a possible nine. In the standard care group, patients received a mean of 8.9 (median 9, range 6-9) injections of pegaptanib. Including the required administration at day 0 , active verteporfin photodynamic therapy was administered a mean of 3.2 times (median 3, range 2-5) in the verteporfin standard care group, and placebo verteporfin was administered a mean of 2.5 times (median 3, 1-5) in the bevacizumab group. The mean number of sham treatments was 7.3 (median 9, range 3-9) in the 12 patients randomised to sham intravitreal injections in the standard care group.

Randomised treatment groups were similar with regard to demographic and baseline ocular and morphological characteristics (table 1).

\section{Primary and secondary end points}

All end points with respect to visual acuity in the study eye at 54 weeks favoured treatment with bevacizumab over standard care. With respect to primary efficacy, 21 patients (32\%, 95\% confidence interval $22 \%$ to $46 \%$ ) in the bevacizumab gained 15 or more letters from baseline visual acuity compared with two (3\%, 0.4\% to $11 \%)$ in the standard care group $(\mathrm{P}<0.001$; odds ratio 18.1 (3.6 to 91.2), adjusted for baseline differences in age, visual acuity, and sex; fig 2 , table 2). The number needed to treat was 4 (3 to 6). Available case analysis gave similar results with respect to the primary efficacy end point; 33\% (21/64) of patients in the bevacizumab group gained 15 or more letters compared with $3 \%(2 / 62)$ in the standard care group $(\mathrm{P}<0.001$; odds ratio 18.4 (3.6 to 93.8), adjusted for baseline differences in age, visual acuity, and sex). There seemed to be a beneficial effect for both lesion subtypes of choroidal neovascularisation but prespecified subgroup analyses such as this should be interpreted with great caution (fig 3). In addition, the proportion of patients who lost fewer than 15 letters of visual acuity from baseline was significantly greater among those receiving bevacizumab treatment than standard care (59 $(91 \%)$ v $44(67 \%), \mathrm{P}<0.001$; odds ratio 4.7 (1.7 to 13.0); fig 2, table 2). The proportion of patients in the bevacizumab who gained 10 or more letters from 
Table $2 \mid$ Primary and key secondary efficacy outcome measures according to gains and losses in letters read on ETDRS chart. Figures are percentages (numbers) of participants

\begin{tabular}{lcccc} 
Vision change & $\begin{array}{c}\text { Standard therapy } \\
(\mathrm{n}=66)^{\star}\end{array}$ & $\begin{array}{c}\text { Bevacizumab } \\
(\mathrm{n}=65) \dagger\end{array}$ & $\begin{array}{c}\text { Difference }(95 \% \mathrm{Cl}), \\
\mathbf{P} \text { value }\end{array}$ & $\begin{array}{c}\text { Adjusted } \neq \text { odds } \\
\text { ratio }(95 \% \mathrm{Cl})\end{array}$ \\
Gain of $\geq 15$ & $3(2)$ & $32(21)$ & $29(17$ to 41$),<0.001$ & $18.1(3.6$ to 91.2$)$ \\
\hline Gain of $\geq 10$ & $8(5)$ & $46(30)$ & $39(25$ to 52$),<0.001$ & $10.8(3.6$ to 32.0$)$ \\
\hline Loss of $<15$ & $67(44)$ & $91(59)$ & $24(11$ to 385$),<0.001$ & $4.7(1.7$ to 13.0$)$ \\
\hline
\end{tabular}

ETDRS=Early Treatment Diabetic Retinopathy Study.

* Last observation carried forward for four patients.

tLast observation carried forward for one patient.

†Adjusted for age, sex, baseline visual acuity.

baseline visual acuity was greater than in the standard care group, odds ratio 8.5 (3.1 to 23.4) (fig 2, table 2).

A greater proportion of patients treated with bevacizumab than standard care had visual acuity of about $6 / 12$ or better and smaller proportions had visual acuity of about $6 / 60$ or worse (fig 4 ). One patient in the bevacizumab group and three patients in the standard care group experienced severe loss of visual acuity (defined as a decrease of 30 letters or more). Although a predefined exploratory subanalysis of the patients randomised to bevacizumab suggests that the primary outcome measure was not dependent on the outcome of a single arm, it must be noted that even prespecified subgroup analyses should be interpreted with great caution. This subgroup analysis (see appendix on bmj.com) showed that the proportion of patients gaining 15 or more letters at week 54 compared with baseline was $44 \%$ with bevacizumab (median letter change 13.5) $v 0 \%$ with photodynamic therapy (change -16.5 ); $30 \%$ with bevacizumab (change 8.0 ) v $5 \%$ with pegaptanib (change -5.5 ), and $25 \%$ with bevacizumab (change 3.0) $v 0 \%$ with sham (change-10.5).

Following the mean changes in visual acuity scores over time showed that the values in the bevacizumab group were superior to those in the standard care group at each six weekly review (fig 5 . On average, visual acuity of patients treated with bevacizumab increased by 6.3 letters at six weeks after the first treatment (week 6 ), and increased slightly further over time to a gain 6.6 letters six weeks after the final loading phase of three injections (week 18) and to 7.0 letters by 54 weeks. In contrast, patients in the standard care group had an average loss in visual acuity at each six weekly follow-up visit, with a mean loss of 9.4 letters by 54 weeks.

Table 3 summarises results for prespecified exploratory end points related to the morphological characteristics of lesions. At final follow-up at 54 weeks, the area occupied by choroidal neovascularisation, as measured by a masked independent reading centre, showed a greater regression in the bevacizumab group compared with the standard care group (median change $\left.-0.9 \mathrm{~mm}^{2} v-0.3 \mathrm{~mm}^{2}\right)$. The area of choroidal neovascularisation leak at 54 weeks decreased by a median of $2.5 \mathrm{~mm}^{2}$ in the bevacizumab group compared with $1.3 \mathrm{~mm}^{2}$ in the standard care group $(\mathrm{P}<0.3)$.

The mean lesion area increased by $3.3 \mathrm{~mm}^{2}$ in the standard care group and by $0.9 \mathrm{~mm}^{2}$ in the bevacizumab group $(\mathrm{P}=0.03)$. Central retinal thickness as measured by optical coherence tomography decreased by a mean of $91 \mu \mathrm{m}$ in the bevacizumab treated group and $55 \mu \mathrm{m}$ in the standard care group $(\mathrm{P}=0.08)$.

\section{Adverse events}

Table 4 summarises the safety results. Serious ocular adverse events associated with bevacizumab were uncommon. There were no cases of endophthalmitis, classified as a condition treated with intravitreal or systemic antibiotics. One patient in the standard care group who received pegaptanib experienced rhegmatogenous retinal detachment. There was a single case of vitreous haemorrhage in the bevacizumab group and no cases in the standard care group.

Rates of adverse events of intraocular inflammation graded as $\geq 1$ (pooled for reported events of iritis, iridocyclitis, vitritis, uveitis, and anterior chamber inflammation) occurred in two patients in the bevacizumab group and one in the standard care group. In the bevacizumab group the two cases of anterior chamber inflammation were due to herpes simplex virus kerato-uveitis (a new presentation in one patient and a recurrence in the other patient), and the case in the standard care/sham arm it was idiopathic. No patients in the bevacizumab group required topical corticosteroids to treat the inflammation. There were no cases of a rise in intraocular pressure requiring treatment after bevacizumab injection, though one patient treated with pegaptanib in the standard care group required treatment after an intravitreal injection.

There were no cases of cataract in the study eye that required surgery in any patient during the study. No one in the bevacizumab group experienced progression of cataract $(>2$ step change in LOCS II grading) compared with $1 / 66$ of those in the standard care group. There were no reports of traumatic cataract in the study eye of any patient during the study.

Three serious non-ocular adverse events occurred in the bevacizumab group (two myocardial infarctions and one atrial fibrillation) and seven in the standard care group (five unexpected admission to hospital,

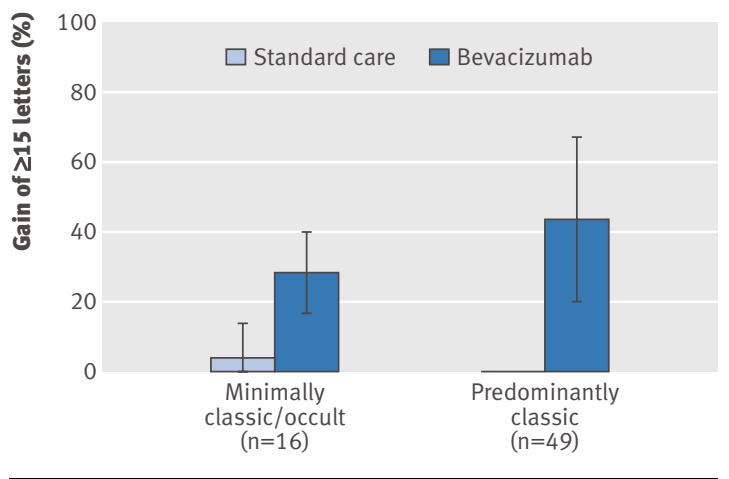

Fig $3 \mid$ Gain of $\geq 15$ letters (moderate gain) from baseline at 54 weeks by type of choroidal neovascularisation lesion. Confidence intervals based on normal approximation and standard errors of proportion. Last observation carried forward used to impute missing data. All statistical tests were two sided. P 0.001 for all comparisons of bevacizumab with standard care 
Table $3 \mid$ Summary of changes from baseline in morphological characteristics of lesions at week 54. Figures are medians (interquartile range)

\begin{tabular}{lcc} 
Treatment end point & Standard care $\left(\mathrm{n}=61^{*}\right)$ & Bevacizumab $(\mathrm{n}=64)$ \\
Classic choroidal neovascularisation $\left(\mathrm{mm}^{2}\right)$ & $0(-1.38-0.06)$ & $-0.26(-1.61-0)$ \\
\hline Choroidal neovascularisation $\left(\mathrm{mm}^{2}\right)$ & $-0.27(-2.58-1.24)$ & $-0.88(-4.08-0.4)$ \\
\hline Lesion size $\left(\mathrm{mm}^{2}\right)$ & $2.33(-0.06-6.44)$ & $-0.03(-1.88-2.63)$ \\
\hline Size of leak from choroidal neovascularisation $\left(\mathrm{mm}^{2}\right)$ & $-1.32(-6.63-0)$ & $-2.45(-8.03-0)$ \\
\hline Central macular thickness $(\mu \mathrm{m}) \dagger$ & $-55(-150-7)$ & $-93.5(-144.5--26)$
\end{tabular}

*One patient could not undergo follow-up assessment at 54 weeks.

†With optical coherence tomography.

\section{DISCUSSION}

Intravitreous bevacizumab treatment is associated with a greater chance of moderate vision recovery and a reduced risk of moderate vision loss and improves mean visual acuity at one year in patients with neovascular AMD compared with standard treatment. In addition, more than $45 \%$ of the patients treated with bevacizumab improved 10 or more letters, a threshold that exceeds the variability of the measurement of visual acuity and represents the proportion of patients recovering vision. ${ }^{16}$

one atrial fibrillation, and one episode of prolonged bleeding after excision of a benign neck tumour). One patient in the bevacizumab group died (myocardial infarction). This patient had a pre-existing history of cardiac failure that was stable for six months before enrolment into the study. There were no deaths in the standard care group. There was an additional report of a non-fatal myocardial infarction in the bevacizumab treated group, though this was based on a rise in cardiac enzyme activity without changes on electrocardiography. The patient wanted to continue treatment with bevacizumab and remained in the study after discussion with the data and safety monitoring committee and the patient's cardiologist. The patient continued on the study on active treatment and to date has had no further cardiovascular events.

Though systemic inhibition of pan vascular endothelial growth factor-A isoform has been associated with the occurrence of non-ocular haemorrhage, there were no reports of such events occurring in the bevacizumab group. There was one case of prolonged bleeding after surgical excision of a benign neck tumour in a patient in the standard care group receiving pegaptanib. There were two cases of paroxysmal atrial fibrillation (one in the bevacizumab treated group and one in the standard care group receiving pegaptanib). In addition there were three serious adverse events reported relating to admission to hospital for investigation and management of solid tumours, and a further two caused by admissions for other causes all in the standard care group (table 4).

In the bevacizumab group there was no increase in mean systolic or diastolic blood pressure at the time points measured and no treatment emergent hypertension, a potential adverse events reflecting systemic vascular endothelial growth factor inhibition. The method of single assessment of seated blood pressure is in keeping with other trials in this specialty and might not detect transient rises in blood pressure.

Serious adverse events of arterial thromboembolism were evaluated with criteria from the Antiplatelet Trialists' Collaboration (APTC), in which an event is defined as a non-fatal myocardial infarction, non-fatal ischaemic stroke, non-fatal haemorrhagic stroke, or death from vascular or unknown causes. ${ }^{15}$ Overall, according to this classification, non-stroke events occurred in two patients in the bevacizumab group and none in the standard care group (table 4).

\section{Strengths and limitations}

Our trial incorporates several novel features in its design. ${ }^{14}$ In contrast with previous multicentre randomised controlled trials, ${ }^{51011}$ we used visual gain as our primary outcome measure rather than stabilisation in vision, with secondary outcomes including mean change in visual acuity to maximise use of data points. visual gain reflects the changing expectation of patients and physicians in the treatment of neovascular AMD.

Treatment in the comparator or standard care arm was determined according to funding of standard treatment. This could be viewed as both a strength and a weakness of the study. Though not allowing comparison with a single agent, the choice of a comparator arm with one of three different treatments (two active and one sham) reflects the usual treatment of patients in the NHS at the time of recruitment. It does not undermine the analysis of results as all treatments in the comparator arm have been shown to offer patients a similar chance of improvement in vision (a maximum proportion of 0.06 improving vision with either comparator
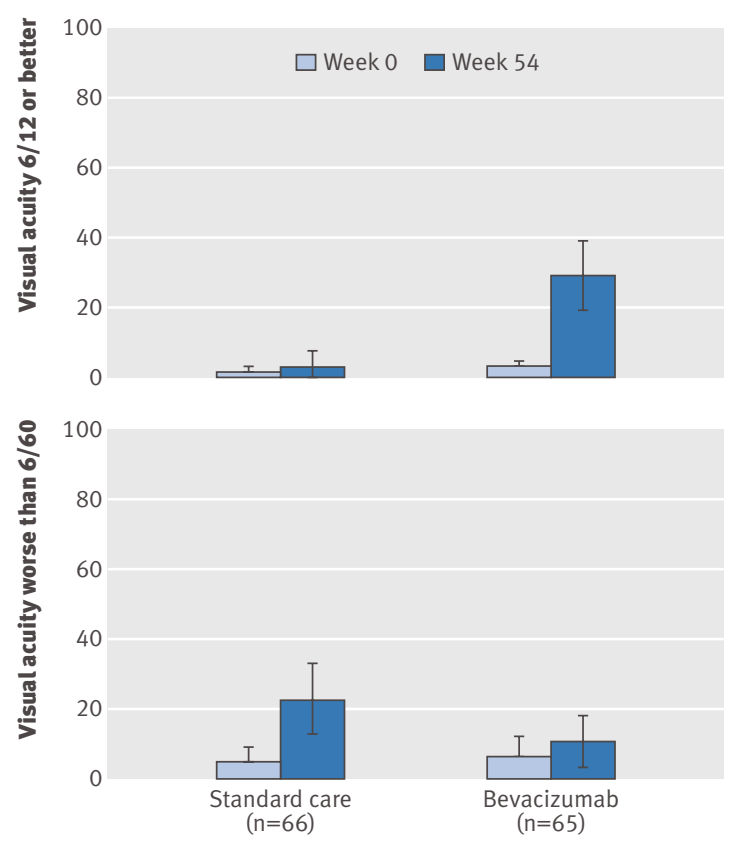

Fig $4 \mid$ Vision of Snellen equivalent of $\geq 6 / 12$ or $\leq 6 / 60$ at week 0 and 54 according to allocation to standard care or bevacizumab This shift in primary end point from stabilisation to 
Table $4 \mid$ Adverse events in patients with neovascular AMD according to treatment. Figures are percentages (numbers) of participants unless specified otherwise

\begin{tabular}{|c|c|c|c|c|c|}
\hline & \multirow[b]{2}{*}{$\begin{array}{c}\text { Bevacizumab } \\
\quad(n=65)\end{array}$} & \multicolumn{4}{|c|}{ Standard care group } \\
\hline & & $\begin{array}{l}\text { Pegaptanib } \\
\quad(n=38)\end{array}$ & $\begin{array}{l}\text { Verteporfin } \\
(n=16)\end{array}$ & $\begin{array}{l}\text { Sham } \\
(n=12)\end{array}$ & $\begin{array}{c}\text { Total } \\
(n=66)\end{array}$ \\
\hline \multicolumn{6}{|l|}{ Serious ocular adverse event } \\
\hline Presumed endophthalmitis & 0 & 0 & 0 & 0 & 0 \\
\hline Uveitis & $3(2)$ & 0 & 0 & $8(1)$ & $2(1)$ \\
\hline $\begin{array}{l}\text { Rhegmatogenous retinal } \\
\text { detachment }\end{array}$ & 0 & $3(1)$ & 0 & 0 & $2(1)$ \\
\hline Retinal tear & 0 & 0 & 0 & 0 & 0 \\
\hline Vitreous haemorrhage & 2(1) & 0 & 0 & 0 & 0 \\
\hline Lens damage & 0 & 0 & 0 & & 0 \\
\hline \multicolumn{6}{|c|}{ Most severe ocular inflammation: } \\
\hline None & $88(57)$ & $97(37)$ & $94(15)$ & $83(10)$ & $94(62)$ \\
\hline Trace & $77(5)$ & $3(1)$ & $6(1)$ & 0 & $3(2)$ \\
\hline$\geq 1$ & $2(1)$ & 0 & 0 & $8(1)$ & $2(1)$ \\
\hline$\geq 2$ & $3(2)$ & 0 & 0 & 0 & 0 \\
\hline$\geq 3$ & 0 & 0 & 0 & $8(1)$ & $2(1)$ \\
\hline$\geq 4$ & 0 & 0 & 0 & 0 & 0 \\
\hline
\end{tabular}

Non-ocular adverse event

\begin{tabular}{llllll}
\hline Treatment emergent hypertension & 0 & 0 & 0 & 0 & 0 \\
\hline
\end{tabular}

Mean change in blood pressure from baseline (systolic/diastolic $\mathrm{mm} \mathrm{Hg}$ ):

\begin{tabular}{llllll} 
Week 1 & $-1.1 /-1.3$ & - & - & - & $-2.2 /-4.8$ \\
\hline Week 54 & $-5.4 /-1.6$ & - & - & - & $-9.2 / 0.14$
\end{tabular}

Key arterial non-fatal thromboembolic events:

\begin{tabular}{lccccc}
\hline Myocardial infarction & $2(1)$ & 0 & 0 & 0 & 0 \\
\hline Stroke & 0 & 0 & 0 & 0 & 0 \\
\hline Cerebral infarction & 0 & 0 & 0 & 0 & 0 \\
\hline
\end{tabular}

Death:

\begin{tabular}{lccccc} 
Vascular cause $^{\star}$ & $2(1)$ & 0 & 0 & 0 & 0 \\
\hline Non-vascular cause & 0 & 0 & 0 & 0 & 0
\end{tabular}

Non-ocular haemorrhage:

\begin{tabular}{llllll}
\hline Any & 0 & 1 & 0 & 0 & 1 \\
\hline $\begin{array}{c}\text { Reported as serious adverse } \\
\text { event }\end{array}$ & 0 & 1 & 0 & 0 & 1
\end{tabular}

*According to Antiplatelet Trialists' Collaboration criteria.

treatment at one year). ${ }^{35} \mathrm{We}$ did not include ranibizumab as one of the treatments included in the comparator arm as this drug reached market only after the start of recruitment and was not widely available during the recruitment phase of this trial.

Another novel design feature is the use of treatment as required with bevacizumab (based on the investigator's decision with standardised retreatment criteria) after three initial treatments. This approach reflects clinical practice, with clinicians using retreatment criteria to determine further treatment with anti-vascular endothelial growth factor agents and should make the results more translatable into clinical practice. This is distinct from the approach taken in other pivotal trials, ${ }^{1011}$ however, which used a continuous treatment strategy not reflected in the EMEA product label or clinical practice. We found no reduction in visual acuity after the loading phase of three bevacizumab injections through to week 54, supporting the algorithm used to determine retreatment.
Intravitreal injections of bevacizumab were associated with a low rate of serious ocular adverse events, including such key events as presumed endophthalmitis, severe intraocular inflammation, and retinal detachment. The ocular safety profiles for the two treatment groups showed no overall imbalance in serious and non-serious ocular adverse events.

Regarding adverse events that potentially reflect systemic inhibition of vascular endothelial growth factor, ${ }^{6}$ we found no imbalance in adverse events between the groups in treatment emergent hypertension or in blood pressure. Patients treated with bevacizumab had a no occurrences of non-ocular haemorrhages, but the rate of arterial thromboembolic events (according to Antiplatelet Trialists' Collaboration criteria) was two in 65 compared with zero in 66 in the standard care group. Both of the thromboembolic events were myocardial infarctions, one in a patient with a history of cardiac failure who developed an infarction after three treatments and one in a patient six months after the start of the study who continued on treatment with bevacizumab with no further cardiovascular events. None of the patients in the bevacizumab group developed cerebrovascular complications, which are thought to occur slightly more commonly with this class of drug treatment for neovascular AMD. ${ }^{17}{ }^{18}$ We could not distinguish small differences in rare adverse events among treatment groups, which would have required large trials of thousands of patients to have sufficient power to detect a small increased risk of a thromboembolic event of around 1/100. To date there have not been any trials of any anti-vascular endothelial growth factor drug given intravitreally that have sufficient power to determine whether there is a small increased risk of thromboembolic events.

Our results provide an unbiased estimate of the efficacy of intravitreal bevacizumab injections for the
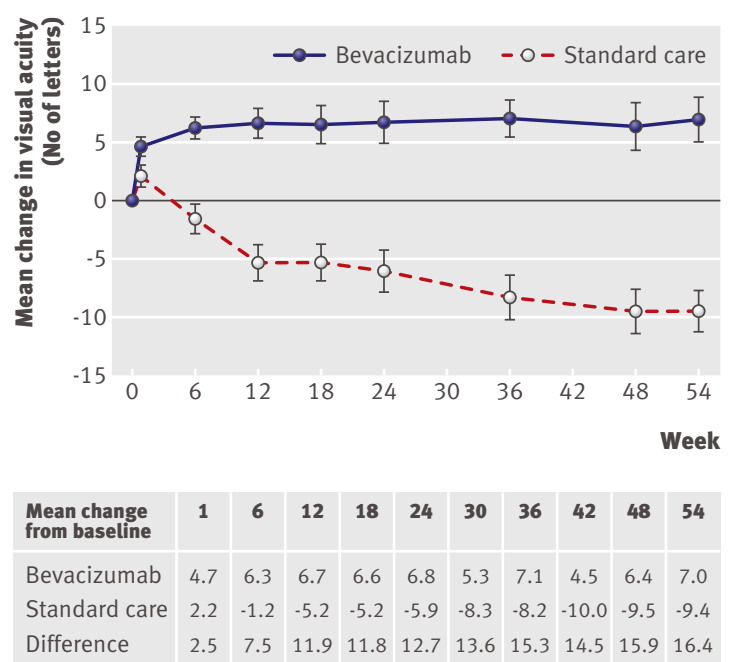

Fig 5 | Mean (SE) changes in number of letters read as measure of refracted visual acuity from baseline to 54 weeks. Best corrected refracted visual acuity obtained at all time points except 30 and 42 weeks, when we obtained unrefracted visual acuity. Last observation carried forward used to impute missing data 


\section{WHAT IS ALREADY KNOWN ON THIS TOPIC}

Anti-vascular endothelial growth factor agents delivered by intravitreous injection are used to treat neovascular age related macular degeneration (AMD)

Bevacizumab has become one of the most widely used agents worldwide, despite being unlicensed and without level 1 evidence for this indication

\section{WHAT THIS STUDY ADDS}

This trial provides level 1 evidence (that is, evidence from at least one properly designed randomised controlled trial) for the use of intravitreous bevacizumab for the treatment of neovascular AMD

A third of patients gained 15 or more letters of visual acuity from baseline compared with $3 \%$ in the standard care group

The results were achieved with visits every six weeks, resulting in a reduction of hospital visits by a third compared with the four weekly cycles used in other trials

treatment of neovascular AMD and support the outcomes of several uncontrolled case $\operatorname{series}^{819-24}$ that have resulted in the widespread off label use of bevacizumab in many countries. Most patients in this study (about three quarters) had the minimally classic-occult subtype of neovascular AMD, the subtype associated with a lower mean change in vision in the pivotal ranibizumab studies. ${ }^{1011}$ The mean change in visual acuity outcomes is in keeping with the outcomes predicted from a systematic review of the published bevacizumab case series and unmasked studies but it was achieved with a less frequent dosing schedule than used in most previous studies. ${ }^{12}$ The continued use of intravitreous bevacizumb has resulted in controversy after the introduction and licensing of ranibizumab, the first licensed treatment for neovascular AMD to result in a mean improvement in visual acuity. One randomised trial examined ranibizumab compared with verteporfin photodynamic therapy ${ }^{10}$ in the predominantly classic form of neovascular AMD, but in its pivotal trial for minimally classic-occult forms of neovascular AMD was compared with sham treatment (not pegaptanib). ${ }^{11}$ No trial exists or is being planned to compare ranibizumab with pegaptanib as it has been presumed that the difference in outcomes in their respective pivotal trials suggests that ranibizumab is superior. Several trials, however, are recruiting to compare ranibizumab with bevacizumab but are unlikely to report for at least 18 months. ${ }^{2526}$ These ongoing trials deliver ranibizumab at its scheduled monthly cycle, but to allow for masking, patients randomised to bevacizumab are also placed on monthly cycles, which negates the potential benefit of the six week clinical effectiveness of bevacizumab ${ }^{27}$ A six weekly review/retreatment cycle with bevacizumab, as supported by our study, reduces the treatment burden by a third for this long term intervention in an elderly target population.

\section{Conclusions and policy implications}

The use of bevacizumab as the main treatment of neovascular AMD outside western Europe and North America and persistence of its use in the US, despite the availability of alternative licensed treatments, is presumed to be because of the lower cost of bevacizumab and is a cause of controversy. ${ }^{28-33}$ Cost effectiveness modelling of bevacizumab in the context of the outcomes of this trial would make this intervention highly cost effective. ${ }^{34}$ The much lower cost and longer duration of action of bevacizumab compared with the only other intervention (ranibizumab) that results in visual recovery for the average patient with neovascular AMD, and its superiority to other licensed treatments as shown in this study, support its immediate implementation in healthcare systems whose budgetary limitations prevent patients' access to ranibizumab. This would have a rapid impact of reducing incident global blindness.

In summary, our trial shows that bevacizumab administered in a six weekly variable retreatment regimen by intravitreous injection for neovascular AMD was superior in efficacy to the standard care available at the start of the trial (verteporfin photodynamic therapy, pegaptanib, or sham). The variable dosing regimen used was effective and not associated with a decline in mean visual acuity after the initial loading phase of three injections. The 54 week results showed that bevacizumab is effective with an acceptable adverse event profile in the treatment of all angiographic subtypes of choroidal neovascularisation secondary to AMD.

Trial investigators: Adnan Tufail (chief investigator); Laura Henderson, Ola Segun-Odumosu (trial managers).

Co-investigators: Praveen J Patel (principal investigator), Catherine Egan, Philip Hykin, Zdenek Gregor, Jonathan Dowler (Moorfields); Mohammed Majid (principal investigator), Clare Bailey (Bristol); Quresh Mohamed (principal investigator), Robert Johnston (Cheltenham)

Recruitment support: Richard Andrews, Andrew Webster. Data management: Richard Seeberan, Catey Bunce, Wen Xing. Statistical analysis: Catey Bunce, Wen Xing

Pharmacy/drug management: Jagdev Bains and Moorfields Pharmacy; Moorfields Pharmaceuticals prepared bevacizumab for intraocular use. Staff in clinical trials unit: Kerry Waller, Felicia Ikeji, Matthew Richardson, Kanom Bibi.

Optometry: Dan Ehrlich, Catherine Grigg, Sonal Rughani, Jen Smith, Shima Shah, Mina Devani, Graham Brown, Rebecca Black.

Treating doctors: Sobha Sivaprasad, Andrew Browning, Yvonne D’Souza, Nachi Acharya, Andrew Kaines, Sam Fraser-Bell, Maria Niskopolou, Noel Horgan, Fred K Chen, Waheeda Rahman, Rajen Gupta, Richard Hanson, Tariq Aslam, Mohammed Musadiq, Tryfon Rotsos, Gayatri Banerjee. Image acquisition: Matthew Richardson, Kulwant Sehmi, Richard Poynter. Image reading centre: Network of Reading Centres, UK: Usha

Chakravarthy, Simon Harding, Nicola Duff, Alison Murphy, Liam Patton, Tunde Peto, Vittorio Silvestri.

Data and safety monitoring committee: Marion Campbell (chair), Frank Holz, Robyn Guymer.

Steering committee: Astrid Fletcher (chair), Catey Bunce, Praveen J Patel, Adnan Tufail, Richard Wormald.

Contributors: AT and PJP were responsible for the study concept and design, with additional input from CE. PJP, CE, LDC, ZG, JD, CB, QM, and RJ contributed to recruitment of patients, data collection, and manuscript review, CB, WX, AT, and PJP undertook analysis and interpretation of the data, AT and PJP drafted the manuscript. AT, PJP, CB, and WX had full access to all of the data in the study and take responsibility for the integrity of the data and the accuracy of the analysis. AT and PJP are guarantors.

Funding: This study was funded by the special trustees of Moorfields Eye Hospital. We also received financial support from the Department of Health through an award made by the National Institute for Health Research to Moorfields Eye Hospital NHS Foundation Trust and UCL Institute of Ophthalmology for a Specialist Biomedical Research Centre 
for Ophthalmology. The views expressed in this publication are those of the authors and not necessarily those of the Department of Health. Additional local support was obtained from the National Eye Research Centre, Bristol.

Role of funding source: The sponsors of the study had no role in study design, data collection, data analysis, data interpretation, or writing of the report.

Competing interests: AT has been on advisory boards for Novartis, Pfizer, GSK, MSD, Allergan. PJP has received travel grants from Novartis. CE has been on advisory boards for Novartis, Pfizer, Allergan. PH has been on advisory boards for Novartis. MM has received travel grants from Novartis. CB has received research grants for investigator sponsored trials, travel grants, and lecture fees from Novartis. RI has received money for speaking engagements from Novartis, and is a shareholder of a software company that has business links with Novartis and Pfizer. The pharmaceutical division at Moorfields (Moorfields Pharmaceuticals) is involved in the repackaging of bevacizumab for intraocular use for sale to other institutions. The authors who work at Moorfields Eye Hospital have no financial gain from this endeavour, and no patents or patent applications with regard bevacizumab are owned by the authors or Moorfields Pharmaceuticals.

Ethical approval: This study was approved by the ethics committees of al clinical centres. Patients provided written informed consent for study participation

Data sharing: No additional data available.

1 Klein R, Klein BE, Jensen SC, Mares-Perlman JA, Cruickshanks KJ, Palta M. Age-related maculopathy in a multiracial United States population: the National Health and Nutrition Examination Survey III. Ophthalmology 1999;106:1056-65.

2 Friedman DS, O'Colmain BJ, Munoz B, Tomany SC, McCarty C, de Jong PT, et al. Prevalence of age-related macular degeneration in the United States. Arch Ophthalmol 2004:122:564-72.

3 Photodynamic therapy of subfoveal choroidal neovascularization in age-related macular degeneration with verteporfin: one-year results of 2 randomized clinical trials-TAP report. Treatment of age-related macular degeneration with photodynamic therapy (TAP) study group. Arch Ophthalmol 1999;117:1329-45.

4 Bressler NM. Photodynamic therapy of subfoveal choroidal neovascularization in age-related macular degeneration with verteporfin: two-year results of 2 randomized clinical trials-TAP report 2. Arch Ophthalmol 2001;119:198-207.

5 Gragoudas ES, Adamis AP, Cunningham ET Jr, Feinsod M, Guyer DR. Pegaptanib for neovascular age-related macular degeneration. $N$ EnglJ Med 2004;351:2805-16.

6 Hurwitz H, Fehrenbacher L, Novotny W, Cartwright T, Hainsworth J, Heim W, et al. Bevacizumab plus irinotecan, fluorouracil, and leucovorin for metastatic colorectal cancer. $N$ Engl I Med 2004;350:2335-42.

7 Rosenfeld PI, Moshfeghi AA, Puliafito CA. Optical coherence tomography findings after an intravitreal injection of bevacizumab (avastin) for neovascular age-related macular degeneration. Ophthalmic Surg Lasers Imaging 2005;36:331-5.

8 Avery RL, Pieramici DJ, Rabena MD, Castellarin AA, Nasir MA, Giust MJ. Intravitreal bevacizumab (avastin) for neovascular agerelated macular degeneration. Ophthalmology 2006;113:363-72.

9 Spaide RF, Laud K, Fine HF, Klancnik JM Jr, Meyerle CB, Yannuzzi LA, et al. Intravitreal bevacizumab treatment of choroidal neovascularization secondary to age-related macular degeneration. Retina 2006;26:383-90.

10 Rosenfeld PJ, Brown DM, Heier JS, Boyer DS, Kaiser PK, Chung CY, et al. Ranibizumab for neovascular age-related macular degeneration. N Engl J Med 2006;355:1419-31.

11 Brown DM, Kaiser PK, Michels M, Soubrane G, Heier JS, Kim RY, et al. Ranibizumab versus verteporfin for neovascular age-related macular degeneration. N Engl J Med 2006;355:1432-44.

12 Schouten IS, La Heij EC, Webers CA, Lundqvist II, Hendrikse F. A systematic review on the effect of bevacizumab in exudative agerelated macular degeneration. Graefes Arch Clin Exp Ophthalmol 2009;247:1-11.
13 Ziemssen F, Grisanti S, Bartz-Schmidt KU, Spitzer MS. Off-label use of bevacizumab for the treatment of age-related macular degeneration: what is the evidence? Drugs Aging 2009;26:295-320.

14 Patel PJ, Bunce C, Tufail A. A randomised, double-masked phase III/ IV study of the efficacy and safety of avastin(R) (bevacizumab) intravitreal injections compared to standard therapy in subjects with choroidal neovascularisation secondary to age-related macular degeneration: clinical trial design. Trials 2008;9:56.

15 Antiplatelet Trialists' Collaboration. Collaborative overview of randomised trials of antiplatelet therapy-l: prevention of death, myocardial infarction, and stroke by prolonged antiplatelet therapy in various categories of patients. BMJ 1994;308:81-106.

16 Patel PJ, Chen FK, Rubin GS, Tufail A. Intersession repeatability of visual acuity scores in age-related macular degeneration. Invest Ophthalmol Vis Sci 2008;49:4347-52.

17 Dafer RM, Schneck M, Friberg TR, Jay WM. Intravitreal ranibizumab and bevacizumab: a review of risk. Semin Ophthalmol 2007;22:201-4

18 Gillies MC, Wong TY. Ranibizumab for neovascular age-related macular degeneration. N Engl J Med 2007;356:748-9.

19 Bashshur ZF, Schakal A, Hamam RN, El Haibi CP, Jaafar RF, Noureddin BN. Intravitreal bevacizumab vs verteporfin photodynamic therapy for neovascular age-related macular degeneration. Arch Ophthalmol 2007;125:1357-61.

20 Giansanti F, Virgili G, Bini A, Rapizzi E, Giacomelli G, Donati MC, et al. Intravitreal bevacizumab therapy for choroidal neovascularization secondary to age-related macular degeneration: 6-month results of an open-label uncontrolled clinical study. Eur J Ophthalmol 2007;17:230-7.

21 Bashshur ZF, Bazarbachi A, Schakal A, Haddad ZA, El Haibi CP, Noureddin BN. Intravitreal bevacizumab for the management of choroidal neovascularization in age-related macular degeneration. Am J Ophthalmol 2006;142:1-9.

22 Lazic R, Gabric N. Intravitreally administered bevacizumab (avastin) in minimally classic and occult choroidal neovascularization secondary to age-related macular degeneration. Graefes Arch Clin Exp Ophthalmol 2007;245:68-73.

23 Pedersen KB, Sjolie AK, Moller F. Intravitreal bevacizumab (avastin) for neovascular age-related macular degeneration in treatment-naive patients. Acta Ophthalmol 2008 Dec 16 (epub ahead of print).

24 Rich RM, Rosenfeld PJ, Puliafito CA, Dubovy SR, Davis JL, Flynn HW Jr, et al. Short-term safety and efficacy of intravitreal bevacizumab (avastin) for neovascular age-related macular degeneration. Retina 2006;26:495-511

25 Center for Preventive Opthalmology and Biostatistics. Comparison of age-related macular degeneration treatments trials. 2009. www. med.upenn.edu/cpob/studies/CATT.shtml.

26 CTEU Bristol. The IVAN trial. 2009. www.ivan-trial.co.uk/.

27 Costa RA, Jorge R, Calucci D, Melo LA Jr, Cardillo JA, Scott IU. Intravitreal bevacizumab (avastin) in combination with verteporfin photodynamic therapy for choroidal neovascularization associated with age-related macular degeneration (IBeVe Study). Graefes Arch Clin Exp Ophthalmol 2007;245:1273-80.

28 Azad R, Chandra P, Gupta R. The economic implications of the use of anti-vascular endothelial growth factor drugs in age-related macular degeneration. Indian J Ophthalmol 2007;55:441-3.

29 Steinbrook R. The price of sight-ranibizumab, bevacizumab, and the treatment of macular degeneration. $N$ Engl J Med 2006;355:1409-12.

30 Wong TY, Liew G, Mitchell P. Clinical update: new treatments for agerelated macular degeneration. Lancet 2007;370:204-6.

31 Rosenfeld P. New treatments for age-related macular degeneration. Lancet 2007;370:1479.

32 Taylor HR, Pezzullo ML, Guymer R. New treatments for age-related macular degeneration. Lancet 2007;370:1481.

33 Wagle AM, Yeo TK, Au Eong KG. New treatments for age-related macular degeneration. Lancet 2007;370:1481.

34 Raftery J, Clegg A, Jones J, Tan SC, Lotery A. Ranibizumab (lucentis) versus bevacizumab (avastin): modelling cost effectiveness. $\mathrm{Br}$ J Ophthalmol 2007;91:1244-6.

Accepted: 15 April 2010 